\title{
Predictors of Atrial Fibrillation in Patients With Cryptogenic Stroke
}

The Neurohospitalist

2019, Vol. 9(3) 127-132

(C) The Author(s) 2018

Article reuse guidelines:

sagepub.com/journals-permissions

DOI: $10.1177 / 1941874418819619$

journals.sagepub.com/home/ $\mathrm{NHO}$

(S)AGE

\author{
Swetha Renati, MD' ${ }^{\mathbb{D}}$, David K. Stone, MD, $\mathbf{P h D}^{2}$, \\ Leonardo Almeida, MD², and Christina A. Wilson, MD, PhD ${ }^{2}$
}

\begin{abstract}
Background and Purpose: Many patients diagnosed with cryptogenic stroke or transient ischemic attack are subsequently found to have atrial fibrillation (AF) on outpatient cardiac telemetry monitoring. Identification of predictive factors for the detection of AF could assist with patient selection to increase the yield of telemetry and hasten initiation of appropriate secondary stroke prevention. Methods: This was a retrospective cross-sectional study of patients diagnosed with cryptogenic stroke at a comprehensive stroke center and referred for at least 21 days of prolonged outpatient telemetry. Telemetry reports and data from the patient's stroke hospitalization, including imaging studies, electrocardiogram (EKG), echocardiogram, vital signs, and laboratory data, were reviewed. Results: Ten percent of the $12 \mathrm{I}$ patients included in the study were diagnosed with AF based on outpatient telemetry. There was a strong association between presence of premature atrial contractions (PACs) on admission EKG and subsequent detection of AF $(P=.004)$. Large left atrial diameter on echocardiogram was correlated with $A F$ detection in males $(P=.024)$. However, there was no association between $A F$ and other echocardiographic measurements. Thyroid-stimulating hormone (TSH) levels were significantly higher in patients with cryptogenic stroke having AF $(P=.008)$, with a TSH greater than $4.20 \mathrm{mIU} / \mathrm{L}$ predictive of detection of $A F(P<.00 \mathrm{I})$. Conclusions: Atrial fibrillation was found by outpatient monitoring in a notable percentage of patients with cryptogenic stroke. Predictors of occult AF in our study population included PACs and higher TSH levels. Although an association between low TSH and AF has been well established, our results suggest that high TSH may be a predictive factor as well.
\end{abstract}

\section{Keywords}

arrhythmia, cerebral embolism and thrombosis, thyroid gland, atrial fibrillation, cerebrovascular disease/stroke

\section{Introduction}

Stroke is the leading cause of functional impairment and the fifth leading cause of death in the United States. ${ }^{1}$ Despite advances in treatment of ischemic stroke, prevention is essential to reduce the morbidity and mortality associated with stroke. Cryptogenic stroke or stroke of undetermined etiology currently accounts for up to one-third of all strokes and can be a diagnostic dilemma. ${ }^{2-6}$

A frequent cause of cryptogenic stroke is thought to be paroxysmal atrial fibrillation (AF) that eludes detection at the time of initial stroke workup. Identification of the underlying arrhythmia is critical, as institution of appropriate anticoagulant therapy can decrease recurrent stroke by up to $60 \%{ }^{7}$ Atrial fibrillation is associated with at least a 4-fold increased stroke risk, ${ }^{8}$ and paroxysmal AF increases the risk of stroke similar to that of sustained AF. ${ }^{9,10}$ The general prevalence of $\mathrm{AF}$ is about $1 \%$ but increases to $9 \%$ in those greater than 80 years of age. ${ }^{1}$ Patients with cryptogenic stroke who underwent outpatient telemetry monitoring were found to have a significant detection rate of $\mathrm{AF}$, ranging from $4 \%$ to $24 \%$, and those who were monitored for longer periods had higher rates of AF detection. ${ }^{11-18}$ Identification of predictors for the detection of $\mathrm{AF}$ could assist with patient selection to increase the yield of telemetry and hasten initiation of appropriate secondary stroke prevention.

We sought to identify the detection rate of AF in patients without identifiable cause of stroke (cryptogenic) who were referred for outpatient cardiac telemetry. We also assessed for association between variables on index admission such as cardiac characteristics, vascular risk factors, and subsequent

\footnotetext{
' Department of Neurology, University of South Florida, Tampa, FL, USA

${ }^{2}$ Department of Neurology, University of Florida, Gainesville, FL, USA

Corresponding Author:

Swetha Renati, Department of Neurology, University of South Florida, 2 Tampa General Circle, 6th Floor, Tampa, FL 33606, USA.

Email: srenati@health.usf.edu
} 
detection of AF during prolonged outpatient cardiac telemetry in this population.

\section{Materials and Methods}

This was a retrospective cross-sectional study of patients diagnosed with cryptogenic stroke or transient ischemic attack (TIA) at a comprehensive stroke center (University of Florida Health) who were subsequently referred to outpatient telemetry (CardioNet; BioTelemetry, Inc, Malvern, Pennsylvania) for arrhythmia monitoring. Patients were monitored with either a CardioNet mobile cardiology outpatient telemetry (MCOT) device or an event monitor, both of which provided automatic arrhythmia detection. Patients were referred for outpatient telemetry based on clinical judgment of the treating vascular neurologist. Decision of which monitor to provide the patient was at the discretion of the CardioNet representative based upon patient insurance coverage. The institutional review board of University of Florida approved the study. The board waived the need for informed consent as the study involved no more than minimal risk.

A total of 242 patients were referred from University of Florida (UF) Health neurology inpatient service and neurology clinics between January 2012 and July 2015. Of these, 121 patients were included according to predetermined inclusion and exclusion criteria. Patients were included if they were determined to have cryptogenic stroke/TIA by the treating vascular neurologist after review of their vascular imaging, telemetry, electrocardiogram (EKG), and echocardiogram to exclude symptomatic significant carotid stenosis, dissection, known AF, mechanical heart valve, cardiac thrombus, cardiac mass, or cardiac vegetation. All patients had vascular imaging to evaluate carotid arteries. One hundred nineteen patients were evaluated with an echocardiogram during hospitalization and the 2 patients remaining had an echocardiogram during the month of index admission which were unremarkable for mechanical heart valve, cardiac thrombus, mass, or vegetation. Those with AF noted on EKG or telemetry were excluded. Patients who did not wear the prescribed cardiac monitor or those who were referred for cardiac monitoring for other diagnoses (not stroke or TIA) were excluded. All outpatient telemetry reports were reviewed. In those patients with an abnormal rhythm noted on cardiac monitoring who were referred to cardiology, the subsequent cardiology notes were also reviewed. Atrial fibrillation was defined according to the CardioNet monitoring algorithm or as determined at discretion of reviewing cardiologist in case of a cardiology referral. There was no minimum cutoff for duration of AF.

The electronic medical record was examined for information regarding each patient's stroke or TIA. Imaging reports, including MRI, MRA, CT, CTA, carotid duplex, and cardiology studies such as echocardiogram and EKG on admission were reviewed. The degree of atrial dilation noted on echocardiography was defined according to the American Society of Echocardiography's Guidelines and Standards Committee and the Chamber Quantification Writing Group (see table in reference for exact dimensions used for each gender). ${ }^{19}$ Initial blood pressure on admission, as well as laboratory studies obtained during their hospitalization including glucose on admission, thyroid-stimulating hormone (TSH), low-density lipoprotein (LDL), vitamin $\mathrm{B} 12$, and hemoglobin $\mathrm{A}_{1 \mathrm{c}}$ $\left(\mathrm{HbA}_{1 \mathrm{c}}\right)$, was reviewed.

\section{Statistical Analysis}

All statistical tests were performed with SPSS statistics (BM Corp, Released 2013, IBM SPSS Statistics for Windows, Version 22.0; IBM Corp, Armonk, New York). Continuous variables are presented as means (standard deviations). Nominal variables are given as counts and proportions. The variables were analyzed with independent samples $t$ test and analysis of variance and proportions with $\chi^{2}$.

\section{Results}

\section{Study Population}

One hundred twenty-one patients were included. Clinical and demographic data for these patients are presented in Table 1. The mean age was 65.8 (11.3) years. There were $53 \%$ males and $47 \%$ females. Seventeen percent of patients had transient neurological symptoms compatible with TIA and $83 \%$ presented with persistent symptoms given the diagnosis of stroke. All patients with stroke had imaging findings compatible with their diagnosis, and $46 \%$ had multiple areas of abnormal restricted diffusion noted on MRI diffusionweighted imaging. Mobile cardiology outpatient telemetry monitoring was used in $62 \%$ of patients, and $38 \%$ had an event monitor. Compliance with monitoring was suboptimal as only $75 \%$ of patients transmitted data for greater than 14 days $(82.7 \%[\mathrm{n}=62]$ with MCOT and $63 \%[\mathrm{n}=29]$ with event monitor), despite all patients being prescribed a monitor for 21 to 30 days.

\section{Detection of $A F$ and Association With Variables}

Ten percent of patients $(\mathrm{n}=12)$ had newly diagnosed AF detected by outpatient monitoring. Those with newly diagnosed AF had a slightly higher mean age (69.0 [6.2] vs 65.4 [11.7] years) but were not found to be statistically significant $(P=.107)$. There were no differences in detection of AF between hospital presentations as TIA (14.3\%) versus stroke $(9 \% ; P=.461)$. A strong association was found between occurrence of premature atrial contractions (PACs) noted on admission $\mathrm{EKG}$ and $\mathrm{AF}$ later diagnosed through cardiac monitoring $(30.0 \%)$ versus those without AF detected (5.1\%; $P=.004)$.

For patients who underwent echocardiography during their hospitalization, no statistically significant association was found between atrial dilatation or wall hypokinesis and 
Table I. Clinical and Demographical Data of Patients Undergoing Outpatient Cardiac Monitoring. ${ }^{a}$

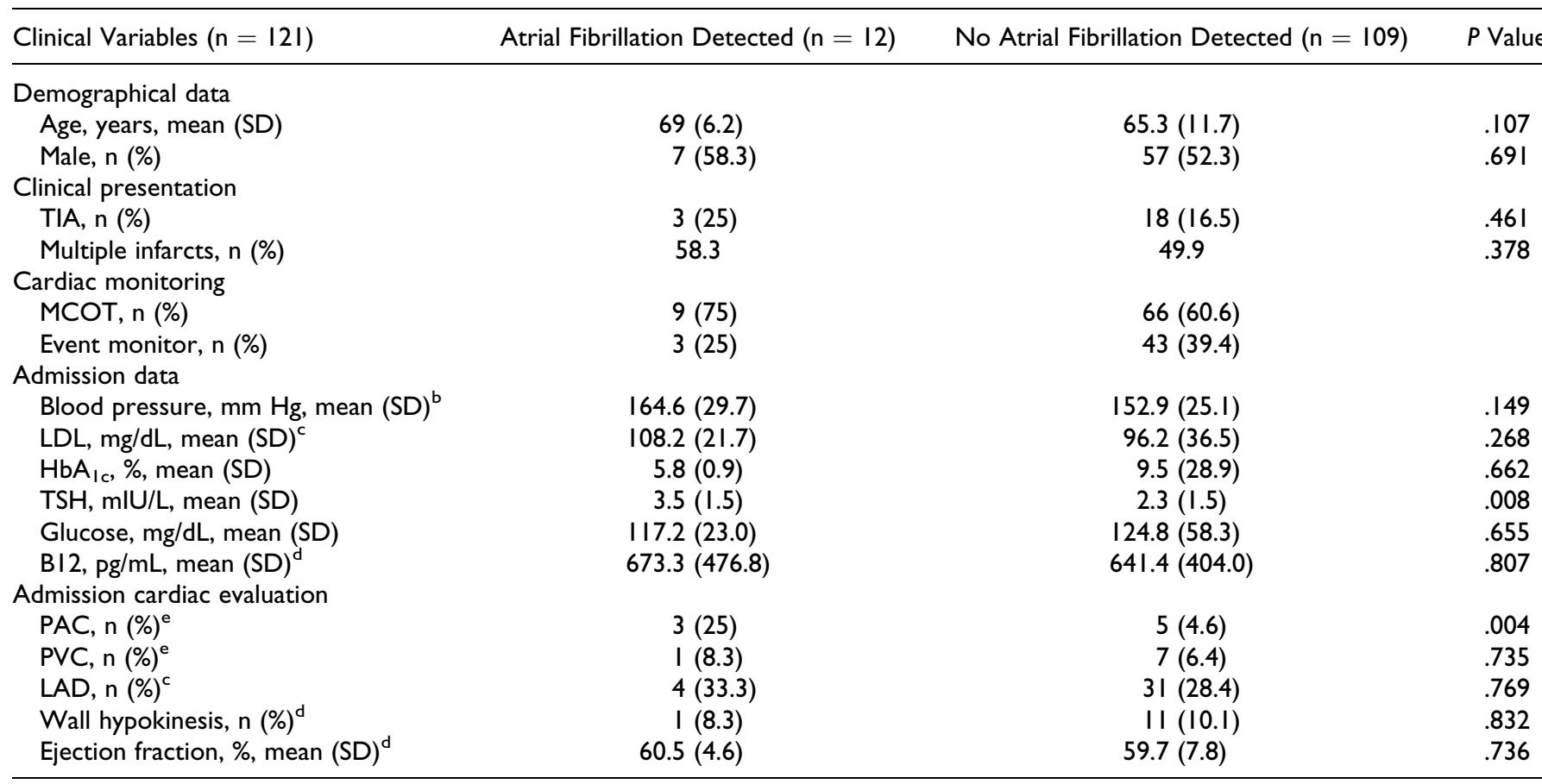

Abbreviations: $\mathrm{HbA}_{I c}$, hemoglobin $\mathrm{A}_{I c}$; LAD, left atrial dilatation; LDL, low-density lipoprotein; MCOT, mobile cardiology outpatient telemetry; PAC, premature atrial contraction; PVC, premature ventricular contraction; TIA, transient ischemic attack; TSH, thyroid-stimulating hormone.

${ }^{\text {a }}$ Percentages are listed in parenthesis.

${ }^{b}$ Data available for only 120 patients.

${ }^{\mathrm{C}}$ Data available for only 118 patients.

${ }^{\mathrm{d} D a t a}$ available for only 119 patients.

${ }^{\mathrm{e}}$ Data available for only 109 patients.

outpatient detection of AF. However, subgroup analysis identified a significant association between enlarged left atrial diameter on echocardiogram and outpatient detection of $\mathrm{AF}$ in males, with $28 \%(\mathrm{n}=2)$ of patients found to have diameters within the range for severe dilatation $(\geq 5.2 \mathrm{~cm})$ in the $\mathrm{AF}$ group versus 0 in the non-AF group $(P=.024)$.

Thyroid-stimulating hormone levels were significantly higher in the AF group than the non-AF group (3.5 [1.5] and 2.3 [1.5], respectively; $P=.008$ ). Normal laboratory range for TSH in our institution is 0.27 to $4.20 \mathrm{mIU} / \mathrm{L}$. When patients were divided into 3 groups (TSH less than 0.27, 0.27-4.20, or greater than 4.2), there was a statistically significant association between TSH $>4.20$ and the detection of $\operatorname{AF}(P<.001)$. Only $10.7 \%$ of total patients fell into the high TSH group (TSH $>$ $4.20 ; \mathrm{n}=13$ ) and yet $46.2 \%$ of those patients were found to have AF during the monitoring period. Whereas no patients in the low TSH group (TSH $<0.27 ; \mathrm{n}=4$ ) and $5.8 \%$ of the patients in the normal TSH range (TSH: 0.27-4.20; $n=104$ ) were found to have AF (Figure 1). Most of the TSH values were in the range for subclinical hypothyroidism, with normal free thyroxine (T4) values noted (Supplemental Table). There were no statistically significant differences between groups in terms of LDL, glucose, $\mathrm{HbA}_{1 \mathrm{c}}$, or B12 levels on admission.

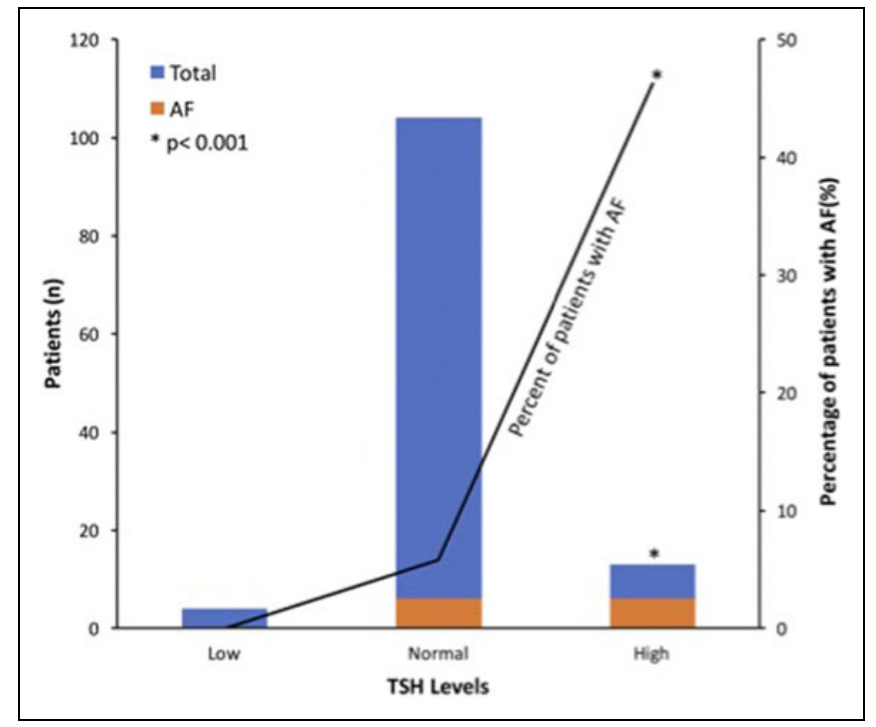

Figure I. Patients with atrial fibrillation (AF) grouped by thyroidstimulating hormone (TSH) levels (low [TSH: < 0.27], normal [TSH: $0.27-4.20]$, high [TSH > 4.2]). There were $46.2 \%$ of patients in the high TSH group who were found to have AF, and there was a significant positive correlation between the 2 variables $(P<.00 \mathrm{I})$. 


\section{Discussion}

Atrial fibrillation was found on outpatient cardiac telemetry in patients initially diagnosed with cryptogenic strokes. As mentioned previously, the detection rate of AF on outpatient cardiac telemetry has been reported to be anywhere from $4 \%$ to $24 \%$. The detection rate in this study was $10 \%$, consistent with previous studies. As would be expected, detection rates improved if telemetry data were transmitted for 14 or more days. Atrial fibrillation detection rate was $12 \%$ in those monitored $\geq 14$ days versus $3.3 \%$ in those monitored $<14$ days, with a trend toward statistical significance $(P=.164)$. There was a trend toward AF being detected more often in older patients, but it was not significant in our study. Of note, most of the patients in this study also underwent in-hospital telemetry and echocardiography, and prior studies have suggested that the rate of detection of AF is lower in those who had previous extensive cardiac imaging. ${ }^{18}$

Premature atrial contractions (PACs), with beats originating outside the sinoatrial node, were long thought to be a benign arrhythmia; however, more recent data suggest that frequent PACs may be associated with increased cardiovascular hospitalization and mortality. ${ }^{20}$ The frequency of PAC occurrence also correlates with a higher risk of $\mathrm{AF}^{20,21}$ Our study confirmed previous reports of PACs as a predictive factor for detection of $\mathrm{AF}$ with cardiac monitoring in those with cryptogenic stroke. ${ }^{13}$ This suggests that presence of PACs in patients with unclear mechanism of stroke may predict those most likely to benefit from prolonged outpatient cardiac monitoring.

Hyperthyroidism has long been known to be associated with AF. ${ }^{22,23}$ However, we found a statistically significant association between higher TSH levels and detection of AF. This suggests that thyroid dysfunctions, both hypothyroidism and hyperthyroidism, may affect the cardiovascular system. Indeed, hypothyroidism is associated with decreased cardiac contractility, decreased cardiac output, ventricular irritability, and coronary artery disease. ${ }^{24}$ Zhang et $\mathrm{al}^{25}$ demonstrated that both hypothyroid and hyperthyroid rats had a significantly increased susceptibility to AF. The original cohort of the Framingham Heart Study patients 60 years of age or older showed that there was a significantly higher rate of AF in those with low thyrotropin levels. However, there was also a nonsignificant trend for those with higher thyrotropin levels. ${ }^{22}$ There have been case reports of patients with subclinical hypothyroidism who developed AF. ${ }^{26}$ In addition, patients with subclinical hypothyroidism were noted to have a higher incidence of postoperative $\mathrm{AF}$ after coronary artery bypass grafting and aortic valve replacement. ${ }^{27,28}$ It should be noted that the overwhelming majority of patients with elevated TSH in our study had subclinical hypothyroidism with normal free T4 levels. Taken together, we suggest a correlation between subclinical hypothyroidism and AF.

As noted above, hypothyroidism is associated with decreased cardiac output and coronary artery disease; these may exert an indirect effect on the atria, predisposing to arrhythmias. Kolettis and Tsatsoulis propose that subclinical hypothyroidism may lead to AF due to the compensated state of thyroid hormones. ${ }^{26}$ They suggest that during this state $\mathrm{T} 4$ is converted to the more active $\mathrm{T} 3$, and the higher T3/T4 ratio may create an environment alike $\mathrm{T} 3$ thyrotoxicosis. ${ }^{26}$

There are reports of an association between subclinical hypothyroidism and certain inflammatory markers (eg, interleukin-6, C-reactive protein, erythrocyte sedimentation rate), ${ }^{29}$ and likewise associations between those inflammatory markers and AF have also been reported. ${ }^{30}$ Although a causal relationship has not been established, it suggests that subclinical hypothyroidism may not be as benign as previously thought, and the association between subclinical hypothyroidism and $\mathrm{AF}$ warrants further study. However, thyroid hormones can also be acute phase reactants and may be elevated in inflammatory states. Our study, found that TSH elevation was correlated with AF detection. Whether this correlation is due to an association with subclinical hypothyroidism or inflammation warrants further study.

Although TIA can be difficult to define, we included TIA in our study as it is our opinion that these patients benefit from monitoring similar to those with cryptogenic stroke. We found no significant difference in the detection of $\mathrm{AF}$ in the group with index admission for TIA versus those with index admission for stroke. Prior studies have also noted detection of occult AF in the cryptogenic TIA population. Tayal et $\mathrm{al}^{11}$ demonstrated the detection of AF by MCOT in cryptogenic TIA or stroke. The Atrial Fibrillation in Patients with Cryptogenic Stroke (EMBRACE) ${ }^{14}$ trial and the Cryptogenic stroke and Underlying Atrial Fibrillation (CRYSTAL AF) ${ }^{31}$ trials also included the cryptogenic TIA population. In the Cryptogenic stroke and Underlying Atrial Fibrillation (CRYSTAL AF trial, $15 \%$ of patients with TIA versus $8.3 \%$ of patients with cryptogenic stroke had AF detected by 6 months with implantable cardiac monitoring. ${ }^{31}$

Although we did not differentiate cortical versus lacunar stroke patterns on imaging in this study, we did identify those with multiple areas of abnormal restricted diffusion versus those with a single focus of restricted diffusion during their index admission. Surprisingly, there was no difference in AF detection between these 2 populations. Although it is more common to observe a cortical pattern, embolic strokes may also appear lacunar. Macdonald et $\mathrm{al}^{32}$ reported that small emboli can enter penetrating vessels resulting in lacunar appearing strokes.

Potential limitations of our study are the relatively low sample size and the retrospective design. This was a singlecenter study conducted at a comprehensive stroke center and thus may not be representative of patients diagnosed with cryptogenic stroke following a more limited workup at a hospital without vascular neurologists. Given lack of standardization in timing of blood draws, it is unclear if thyroid hormones were affected as acute phase reactants. Other limitations 
include the lack of follow-up thyroid hormones in the outpatient setting; this is an area of interest for our future studies. In addition, referral for long-term telemetry monitoring after ruling out prior mentioned causes was based on discretion of the treating neurologist whose own biases could potentially have an unknown influence on our data.

Prior work by others identified older age, PACs, and evidence of prior cortical or cerebellar strokes on imaging to be independent predictors of $\mathrm{AF}$ in patients with cryptogenic stroke. ${ }^{13,15}$ In our study, presence of PACs was also found to be associated with AF. Of note, the work by Favilla and colleagues did not identify any echocardiographic findings to be predictors of AF, including left atrial size. ${ }^{15}$ However, in our cohort, we found severe left atrial enlargement $(\geq 5.2 \mathrm{~cm})$, to be a risk factor for AF only in men. The effect of gender, severity of atrial dilatation, or possible differences in the technique in estimation of left atrial size between the 2 institutions/cardiologist may be considered as potential explanations for the different findings. Indeed, the degree of atrial dilation, or left atrial diameter, was shown in several studies to be positively associated with risk of $\mathrm{AF}^{33-35}$ Other potential markers of atrial cardiopathy (eg, $\mathrm{P}$ terminal force or ProBNP) may be considered in future prospective studies but were not available in this retrospective study.

In conclusion, AF is found in a significant portion of those who were initially labeled as cryptogenic stroke. Evaluation for occult arrhythmia is indicated in these patients, as it is a highly modifiable risk factor. Premature atrial contractions and elevated TSH were noted to be predictive factors for the subsequent development of AF.

\section{Declaration of Conflicting Interests}

The authors declared no potential conflicts of interest with respect to the research, authorship, and/or publication of this article.

\section{Funding}

The authors received no financial support for the research, authorship, and/or publication of this article.

\section{ORCID iD}

Swetha Renati, MD (D) https://orcid.org/0000-0001-6479-3634

\section{Supplemental Material}

Supplemental material for this article is available online.

\section{References}

1. Go AS, Mozaffarian D, Roger VL, et al. Heart disease and stroke statistics - 2014 update: a report from the american heart association. Circulation. 2014;129(3):e28-e292.

2. Grau AJ, Weimar C, Buggle F, et al. Risk factors, outcome, and treatment in subtypes of ischemic stroke: the german stroke data bank. Stroke. 2001;32(11):2559-2566.

3. Putaala J, Metso AJ, Metso TM, et al. Analysis of 1008 consecutive patients aged 15 to 49 with first-ever ischemic stroke: the helsinki young stroke registry. Stroke. 2009;40(4):1195-1203.
4. Li L, Yiin GS, Geraghty OC, et al. Incidence, outcome, risk factors, and long-term prognosis of cryptogenic transient ischaemic attack and ischaemic stroke: a population-based study. Lancet Neurol. 2015;14(9):903-913.

5. Nouh A, Hussain M, Mehta T, Yaghi S. Embolic strokes of unknown source and cryptogenic stroke: implications in clinical practice. Front Neurol. 2016;7:37.

6. Hart RG, Catanese L, Perera KS, Ntaios G, Connolly SJ. Embolic stroke of undetermined source: a systematic review and clinical update. Stroke. 2017;48(4):867-872.

7. Hart RG, Pearce LA, Aguilar MI. Meta-analysis: antithrombotic therapy to prevent stroke in patients who have nonvalvular atrial fibrillation. Ann Intern Med. 2007;146(12):857-867.

8. Kannel WB, Benjamin EJ. Status of the epidemiology of atrial fibrillation. Med Clin North Am. 2008;92(1):17-40, ix

9. Marini C, De Santis F, Sacco S, et al. Contribution of atrial fibrillation to incidence and outcome of ischemic stroke: results from a population-based study. Stroke. 2005;36(6):1115-1119.

10. Hohnloser SH, Pajitnev D, Pogue J, et al. Incidence of stroke in paroxysmal versus sustained atrial fibrillation in patients taking oral anticoagulation or combined antiplatelet therapy: an ACTIVE W substudy. J Am Coll Cardiol. 2007;50(22): 2156-2161.

11. Tayal AH, Tian M, Kelly KM, et al. Atrial fibrillation detected by mobile cardiac outpatient telemetry in cryptogenic tia or stroke. Neurology. 2008;71(21):1696-1701.

12. Bhatt A, Majid A, Razak A, Kassab M, Hussain S, Safdar A. Predictors of occult paroxysmal atrial fibrillation in cryptogenic strokes detected by long-term noninvasive cardiac monitoring. Stroke Res Treat. 2011;2011:172074.

13. Miller DJ, Khan MA, Schultz LR, et al. Outpatient cardiac telemetry detects a high rate of atrial fibrillation in cryptogenic stroke. J Neurol Sci. 2013;324(1-2):57-61.

14. Gladstone DJ, Spring M, Dorian P, et al. Atrial fibrillation in patients with cryptogenic stroke. N Engl J Med. 2014;370: 2467-2477.

15. Favilla CG, Ingala E, Jara J, et al. Predictors of finding occult atrial fibrillation after cryptogenic stroke. Stroke. 2015;46(5): 1210-1215.

16. Stahrenberg R, Weber-Krüger M, Seegers J, et al. Enhanced detection of paroxysmal atrial fibrillation by early and prolonged continuous holter monitoring in patients with cerebral ischemia presenting in sinus rhythm. Stroke. 2010;41(12):2884-2888.

17. Barthélémy JC, Féasson-Gérard S, Garnier P, et al. Automatic cardiac event recorders reveal paroxysmal atrial fibrillation after unexplained strokes or transient ischemic attacks. Ann Noninvasive Electrocardiol. 2003;8(3):194-199.

18. Kalani R, Bernstein R, Passman R, Curran Y, Ruff I, Prabhakaran S. Low yield of mobile cardiac outpatient telemetry after cryptogenic stroke in patients with extensive cardiac imaging. J Stroke Cerebrovasc Dis. 2015;24(9):2069-2073.

19. Lang RM, Bierig M, Devereux RB, et al. Recommendations for chamber quantification: a report from the american society of echocardiography's guidelines and standards committee and the chamber quantification writing group, developed in conjunction 
with the european association of echocardiography, a branch of the european society of cardiology. J Am Soc Echocardiogr. 2005;18(12):1440-1463.

20. Lin CY, Lin YJ, Chen YY, et al. Prognostic significance of premature atrial complexes burden in prediction of long-term outcome. J Am Heart Assoc. 2015;4(9):e002192.

21. Chong BH, Pong V, Lam KF, et al. Frequent premature atrial complexes predict new occurrence of atrial fibrillation and adverse cardiovascular events. Europace. 2012;14(7): 942-947.

22. Sawin CT, Geller A, Wolf PA, et al. Low serum thyrotropin concentrations as a risk factor for atrial fibrillation in older persons. N Engl J Med. 1994;331(19):1249-1252.

23. Gammage MD, Parle JV, Holder RL, et al. Association between serum free thyroxine concentration and atrial fibrillation. Arch Intern Med. 2007;167(9):928-934.

24. Klein I, Danzi S. Thyroid disease and the heart. Circulation. 2007;116:1725-1735.

25. Zhang Y, Dedkov EI, Teplitsky D, et al. Both hypothyroidism and hyperthyroidism increase atrial fibrillation inducibility in rats. Circ Arrhythm Electrophysiol. 2013;6(5):952-959.

26. Kolettis TM, Tsatsoulis A. Subclinical hypothyroidism: an overlooked cause of atrial fibrillation? J Atr Fibrillation. 2012;5(4):710.

27. Park YJ, Yoon JW, Kim KI, et al. Subclinical hypothyroidism might increase the risk of transient atrial fibrillation after coronary artery bypass grafting. Ann Thorac Surg. 2009;87(6): 1846-1852.
28. Martínez-Comendador J, Marcos-Vidal JM, Gualis J, et al. Subclinical hypothyroidism might increase the risk of postoperative atrial fibrillation after aortic valve replacement. Thorac Cardiovasc Surg. 2016;64(5):427-433.

29. Gupta G, Sharma P, Kumar P, Itagappa M. Study on subclinical hypothyroidism and its association with various inflammatory markers. J Clin Diagn Res. 2015;9(11):BC04-BC06.

30. Korantzopoulos P, Letsas KP, Tse G, Fragakis N, Goudis CA, Liu T. Inflammation and atrial fibrillation: a comprehensive review. J Arrhythm. 2018;34(4):394-401.

31. Sanna T, Diener HC, Passman RS, et al. Cryptogenic stroke and underlying atrial fibrillation. $N$ Engl J Med. 2014;370(26): 2478-2486.

32. Macdonald RL, Kowalczuk A, Johns L. Emboli enter penetrating arteries of monkey brain in relation to their size. Stroke. 1995; 26(7):1247-1250; discussion 1250-1241.

33. Zhuang J, Wang Y, Tang K, et al. Association between left atrial size and atrial fibrillation recurrence after single circumferential pulmonary vein isolation: a systematic review and meta-analysis of observational studies. Europace. 2012;14(5): 638-645.

34. Tsang TS, Barnes ME, Bailey KR, et al. Left atrial volume: important risk marker of incident atrial fibrillation in 1655 older men and women. Mayo Clin Proc. 2001;76(5):467-475.

35. Tsang TS, Barnes ME, Gersh BJ, Bailey KR, Seward JB. Risks for atrial fibrillation and congestive heart failure in patients $>/$ $=65$ years of age with abnormal left ventricular diastolic relaxation. Am J Cardiol. 2004;93(1):54-58. 